\title{
Therapeutics
}

\section{A computer based decision support system and risk chart did not reduce cardiovascular risk or blood pressure in hypertension}

\section{Montgomery AA, Fahey T, Peters TJ, et al. Evaluation of computer based clinical decision support system and risk chart for management of hypertension in primary care: randomised controlled trial. BMJ 2000 Mar 11;320:686-90.}

\section{Question}

In patients with hypertension, do a computer based clinical decision support system (CDSS) and a cardiovascular risk chart help to reduce absolute cardiovascular risk and blood pressure?

\section{Design}

Cluster randomised (allocation concealed*), unblinded,* controlled trial with 1 year follow up.

\section{Setting}

27 general practices in Avon, UK.

\section{Patients}

614 patients who were $60-80$ years of age (mean age 71 y, $54 \%$ women), had a diagnosis of hypertension, and were prescribed antihypertensive drugs in the previous year. Follow up was $86 \%$ at 1 year.

\section{Intervention}

General practices were allocated to a CDSS plus a risk chart (10 practices, 229 patients); a risk chart only (10 practices, 228 patients); or usual care (7 practices, 157 patients). The CDSS required patient information to be entered; the patient's 5 year absolute risk for a cardiovascular event was calculated and presented numerically. The risk chart showed a colour coded grid of the ranges of absolute risks for cardiovascular events corresponding to patient risk factors.

\section{Main outcome measures}

The proportion of patients with 5 year cardiovascular risk $\geqslant 10 \%$. Secondary outcomes included blood pressure concentrations.

\section{Main results}

Analysis was by intention to treat. After adjustment for type of computer system and baseline absolute risk, more patients in the CDSS group than in the chart only group were at high risk for a cardiovascular event at 12 months $(p=0.02)$; no differences existed between the intervention groups and usual care (table). After adjustment for type of computer system and baseline blood pressure, the chart only group had a lower mean systolic blood pressure than the usual care group (mean difference $4.6 \mathrm{~mm} \mathrm{Hg}, 95 \%$ CI 0.8 to $8.4, \mathrm{p}=0.02$ ).

\section{Conclusions}

In patients with hypertension, a computer based clinical decision support system did not reduce the 5 year absolute risk for cardiovascular events or increased blood pressure concentrations. The use of a risk chart alone reduced systolic blood pressure.

*See glossary.

Source of funding: NHS Wales Office of Research and Development.

For correspondence: Dr T Fahey, Division of Primary Health Care, University of Bristol,

Bristol BS8 2PR

England, UK. Fax +44 (0)1179287340.

Computer based decision support system plus risk chart (CDSS), risk chart alone, and usual care for hypertension

\begin{tabular}{lllll}
$\begin{array}{l}\text { Outcome at } 12 \\
\text { months }\end{array}$ & Comparisons & $\begin{array}{l}\text { Event } \\
\text { rates }\end{array}$ & RRI (95\% CI) & NNH (CI) \\
$\begin{array}{lllll}\text { At high risk for } \\
\text { cardiovascular events }\end{array}$ & CDSS $v$ chart & $89 \% v 85 \%$ & $9 \%(1$ to 13$)$ & $13(9$ to 86$)$ \\
\cline { 2 - 5 } & CDSS $v$ usual care & $89 \% v 88 \%$ & $5 \%(-5$ to 10$)$ & Not significant \\
& & & RRR (CI) & NNT \\
\cline { 2 - 5 } & Chart $v$ usual care & $85 \% v 88 \%$ & $5 \%(-5$ to 8$)$ & Not significant \\
\hline
\end{tabular}

tAbbreviations defined in glossary; RRI, RRR, NNH, NNT, and $\mathrm{Cl}$ calculated from adjusted odds ratios in article. Adjustment was made for computer system and baseline cardiovascular risk.

\section{COMMENTARY}

CDSSs facilitate the integration of health related information for individuals with an underlying knowledge base so that patient specific recommendations can be provided to healthcare practitioners to assist with their decision making. These systems are beneficial when used to facilitate preventive healthcare delivery and to assist with the dosing of medications with narrow therapeutic windows. ${ }^{1}$ The potential for these systems to improve outcomes in other aspects of health care, however, remains unclear: some studies have shown a benefit while others have not.

The study by Montgomery et al is an important contribution to this area. The trial was well designed and included clinically important end points. The study also included a non-computer based decision aid that was used by itself in 1 of the study groups. The results, including the finding that the CDSS did not lead to improved cardiovascular risk profiles or blood pressure control and that a simple cardiovascular risk chart used alone led to an improvement in systolic blood pressure, have implications for both clinical practice and future research on CDSSs. For clinicians, it is clear that CDSSs designed to assist with the management of complex medical conditions still require additional development and evaluation before being introduced into routine clinical practice. Inexpensive, non-computer based approaches that can be easily integrated into a clinic should also be considered. For researchers, increasing efforts should now focus on identifying those factors that predict whether a CDSS will have a clinically important effect when introduced into clinical practice.

Dereck L Hunt, MD

McMaster University, Hamilton Ontario, Canada

1 Hunt DL, Haynes RB, Hanna SE, et al. Effects of computer-based clinical decision support systems on physician performance and patient outcomes: a systematic review. JAMA1998;280:1339-46. 